\title{
Retraction Note to: Spectrum of cosmological correlation from vacuum fluctuation of stringy axion in entangled de Sitter space
}

\author{
Sayantan Choudhury ${ }^{1, \mathrm{a}}{ }_{\mathbb{D}}$, Sudhakar Panda $^{2,3, \mathrm{~b}}$ \\ ${ }^{1}$ Quantum Gravity and Unified Theory and Theoretical Cosmology Group, Max Planck Institute for Gravitational Physics (Albert Einstein Institute), \\ Am Mühlenberg 1, Golm, 14476 Potsdam, Germany \\ ${ }^{2}$ National Institute of Science Education and Research, Jatni, Bhubaneswar, Odisha 752050, India \\ ${ }^{3}$ Homi Bhabha National Institute, Training School Complex, Anushakti Nagar, Mumbai 400085, India
}

Published online: 1 April 2020

(C) The Author(s) 2020

Retraction to: Eur. Phys. J. C

https://doi.org/10.1140/epjc/s10052-019-7582-x

This article [1] has been retracted due to ethical reasons (duplicate simultaneous submission to another journal).

\section{Reference}

1. S. Choudhury, S. Panda, Spectrum of cosmological correlation from vacuum fluctuation of stringy axion in entangled de Sitter space. Eur. Phys. J. C 80, 67 (2020). https://doi.org/10.1140/epjc/ s10052-019-7582-x

Open Access This article is distributed under the terms of the Creative Commons Attribution 4.0 International License (http://creativecomm ons.org/licenses/by/4.0/), which permits unrestricted use, distribution, and reproduction in any medium, provided you give appropriate credit to the original author(s) and the source, provide a link to the Creative Commons license, and indicate if changes were made.

Funded by SCOAP ${ }^{3}$.

The original article can be found online at https://doi.org/10.1140/ epjc/s10052-019-7582-x.

a e-mails: sayantan.choudhury@aei.mpg.de; sayanphysicsisi@gmail.com (corresponding author)

bE-mail: panda@niser.ac.in 